ARTICLE

Received 20 May 2014 | Accepted 13 Jan 2015 | Published 17 Feb 2015 DOl: 10.1038/ncomms7281

\title{
The polymeric mucin Muc5ac is required for allergic airway hyperreactivity
}

Christopher M. Evans', Dorota S. Raclawska, Fani Ttofali ${ }^{1}$, Deborah R. Liptzin², Ashley A. Fletcher ${ }^{1}$, Daniel N. Harper ${ }^{1}$, Maggie A. McGing ${ }^{1}$, Melissa M. McElwee ${ }^{3}$, Olatunji W. Williams ${ }^{4}$, Elizabeth Sanchez ${ }^{3}$, Michelle G. Roy ${ }^{3}$, Kristen N. Kindrachuk ${ }^{5}$, Thomas A. Wynn ${ }^{5}$, Holger K. Eltzschig ${ }^{6}$, Michael R. Blackburn ${ }^{7}$, Michael J. Tuvim ${ }^{3}$, William J. Janssen ${ }^{1,8}$, David A. Schwartz ${ }^{1} \&$ Burton F. Dickey ${ }^{3}$

In asthma, airflow obstruction is thought to result primarily from inflammation-triggered airway smooth muscle (ASM) contraction. However, anti-inflammatory and smooth musclerelaxing treatments are often temporary or ineffective. Overproduction of the mucin MUC5AC is an additional disease feature that, while strongly associated pathologically, is poorly understood functionally. Here we show that Muc5ac is a central effector of allergic inflammation that is required for airway hyperreactivity ( $A H R$ ) to methacholine (MCh). In mice bred on two well-characterized strain backgrounds ( $\mathrm{C} 57 \mathrm{BL} / 6$ and $\mathrm{BALB} / \mathrm{C}$ ) and exposed to two separate allergic stimuli (ovalbumin and Aspergillus extract), genetic removal of Muc5ac abolishes AHR. Residual MCh responses are identical to unchallenged controls, and although inflammation remains intact, heterogeneous mucous occlusion decreases by $74 \%$. Thus, whereas inflammatory effects on ASM alone are insufficient for AHR, Muc5ac-mediated plugging is an essential mechanism. Inhibiting MUC5AC may be effective for treating asthma and other lung diseases where it is also overproduced.

\footnotetext{
${ }^{1}$ Department of Medicine, University of Colorado School of Medicine, 12700 E 19th Avenue, Mailstop 8611, Research Complex 2, Room 3121, Aurora, Colorado 80045, USA. ${ }^{2}$ Department of Pediatrics, University of Colorado School of Medicine, 13123 East 16th Avenue, B-395, Aurora, Colorado 80045, USA.

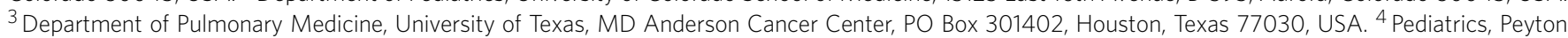
Manning Children's Hospital, 8402 Harcourt Road, Suite 731, Indianapolis, Indiana 46260, USA. ${ }^{5}$ Immunopathogenesis Section, Program in Barrier Immunity and Repair, Laboratory of Parasitic Diseases, National Institute of Allergy and Infectious Diseases, National Institutes of Health, Room 4071, MSC 6606, Bethesda, Maryland 20892-6606, USA. ${ }^{6}$ Department of Anesthesiology, University of Colorado School of Medicine, 12700 E 19 th Avenue, Mailstop B112, Research Complex 2, Room 7124, Aurora, Colorado 80045, USA. ${ }^{7}$ Department of Biochemistry and Molecular Biology, The University of Texas-Houston Medical School, 6431 Fannin, Houston, Texas 77030, USA. ${ }^{8}$ Department of Medicine, National Jewish Health, 1400 Jackson Street, Denver, Colorado 80206 , USA. Correspondence and requests for materials should be addressed to C.M.E. (email: Christopher.Evans@ucdenver.edu).
} 
A sthma is a major health problem. Worldwide, it affects more than 300 million people. In the United States, it affects $\sim 10$ million and costs $\$ 50-60$ billion per year to treat. Asthma is characterized by airflow obstruction and structural changes associated with acute and chronic lung inflammation. Accordingly, the most widely used therapies are bronchodilators and anti-inflammatory agents ${ }^{1}$. However, treatment results are often temporary or incomplete, and alternative strategies are lacking. We propose that this is due to an incomplete understanding of the mechanisms by which airflow obstruction and symptoms of dyspnoea, cough and wheezing are caused by the combined effects of inflammation, bronchoconstriction and additional non-contractile mechanisms. Non-contractile factors include airway wall thickening due to oedema and structural remodelling ${ }^{2}$, loss of airway liquid surface tension due to plasma extravasation and fibrin accumulation $^{3}$ and airway occlusion due to mucus hypersecretion ${ }^{4}$. With even just a small amount of airway smooth muscle (ASM) constriction, these non-contractile components can geometrically amplify airway resistance and increase the propensity for airways to close ${ }^{5-8}$.

In human asthma and in animal models, mucin overproduction and hypersecretion are prominent pathological findings ${ }^{9-14}$. In its most severe setting-fatal asthma-autopsy studies show that patients die from severe airflow obstruction caused by bronchoconstriction coupled with widespread mucous plugging ${ }^{15-17}$. Even in mild to moderate asthma, where there is minimal baseline airflow limitation, patients respond to the inhaled cholinergic agonist methacholine (MCh) with exaggerated obstruction. Existing dogma labels this airway hyperreactivity (AHR) to $\mathrm{MCh}$ as resulting from excessive ASM contraction ${ }^{1}$. Thus, unlike fatal asthma, where mucus hypersecretion is well recognized, in non-fatal exacerbations the potential obstructive role of mucus has been largely overlooked ${ }^{1}$. In fact, in mild to moderate disease, mucin overproduction is highly prevalent ${ }^{12}$, and the genomic region on chromosome $11 \mathrm{p} 15$ that encodes airway polymeric mucins is associated with AHR ${ }^{18}$. Furthermore, mucin overproduction and changes in its biochemical/biophysical properties correlate with asthma exacerbations $4,19,20$. These associations in humans are supported in animal models where mucin overproduction and AHR to MCh are both induced, and where in addition to causing ASM contraction, $\mathrm{MCh}$ is also known to cause mucin secretion ${ }^{21,22}$. Thus, the obstructive effects of ASM contraction may be amplified by acute thickening of mucus on airway surfaces. While these associations are strong, establishing the causative significance of mucus secretion in asthma has been hampered by a lack of experimental evidence and the absence of effective clinical interventions that target the chief glycoprotein components of airway mucus, the secreted polymeric mucins MUC5AC and MUC5B.

MUC5B predominates in healthy human lungs, and its levels in asthma remain stable or in some cases decrease ${ }^{10,12,23}$. On the other hand, MUC5AC levels in asthma increase significantly and consistently $y^{4,10,12,13}$. Mice display similar tightly controlled expression patterns: High baseline $M u c 5 b$ gene expression remains stable following antigen challenge while low baseline Muc5ac gene expression increases markedly ${ }^{24}$. Furthermore, whereas Muc5b is required for airway homeostasis and antibacterial defence, Muc5ac is not essential for these functions ${ }^{25}$, and in lung injury Muc5ac is actually detrimental ${ }^{26}$. These findings implicate Muc5ac as a deleterious and dispensable glycoprotein component of airway mucus. Thus, it is suitable to investigate as a potential target for reducing obstruction in asthma.

In allergic asthma, a biphasic response occurs after allergen inhalation. Acutely, $\operatorname{IgE}$ crosslinking induces mast cell and basophil histamine release, causing a brief episode of airflow obstruction (what is most commonly thought of as an 'asthma attack'). The acute early-phase response lasts a few minutes to a few hours, and it is followed by a late-phase response that lasts for several hours to several days. Clinically, the late phase is considered to be the main driver of disease progression ${ }^{27}$. Accordingly, even after baseline lung function is restored following an acute asthma episode, the ensuing late phase displays sustained pathophysiology hallmarked by AHR to inhaled agonists such as MCh, inflammation characterized by recruitment of eosinophils and interleukin (IL)-13-producing type-2 $\mathrm{T}$ helper cells, and airway remodelling linked to induced MUC5AC production ${ }^{28}$.

MUC5AC/Muc5ac induction is a well-characterized epithelial response to allergic (type 2) inflammation. IL-13 has been implicated in the pathogenesis of human asthma ${ }^{29}$ and in animal models ${ }^{30,31}$. However, compared with its reported effects on leukocytes ${ }^{32}$ or $\mathrm{ASM}^{33}$, the relative importance of IL-13 signalling in epithelia ${ }^{34}$ and the mechanisms by which it elicits asthma-like pathophysiology are unclear. MUC5AC/Muc5ac gene expression is induced by IL-13 through direct stimulation of airway epithelial cells ${ }^{24,30,31,34-36}$. In mice, Muc5ac expression is central to the allergic mucous phenotype. It is induced maximally $48-72 \mathrm{~h}$ after antigen challenge ${ }^{24}$, times that are concordant with late-phase human asthma. We thus hypothesized that eliminating this type- 2 inflammation effector would protect against asthma-like disease in mice.

Here we show the functional significance of Muc5ac in allergic AHR, inflammation and mucus obstruction. In $M u c 5 a c^{-1-}$ mice ${ }^{37}$, we demonstrate that even in the presence of ongoing inflammation, significantly reduced heterogeneous mucous plugging abolishes AHR.

\section{Results}

Muc5ac is a major polymeric mucin in allergic mouse lungs. In wild-type (WT) mice, immunohistochemical analyses demonstrated constitutive Muc5b production that was similar in healthy and ovalbumin (OVA)-sensitized and -challenged airways (Fig. 1a,b). OVA challenge induced Muc5ac protein and alcian blue-periodic acid Schiffs positive glycoconjugate production (Fig. 1a,b), which were marked by a $\sim 400$-fold increase in Muc5ac transcripts (Fig. 1c). In OVA-challenged Muc5ac -/mice, Muc5ac transcripts were absent (Fig. 1c), and bronchial epithelial mucin content was significantly reduced (Fig. 1d,e). We thus examined the development of allergic asthma-like phenotypes in $\mathrm{Muc5ac}^{-1-}$ mice.

Muc5ac is required for AHR to MCh in allergic mouse lungs. To determine the consequences of disrupting Muc5ac expression on airflow obstruction, we assessed lung mechanical function and AHR to MCh. OVA challenge did not alter baseline lung resistance (Supplementary Fig. 1a), demonstrating that inflammatory and mucous metaplastic changes did not disrupt steady-state airflow measurably in this model of late-phase asthma. However, when exposed to MCh, OVA-challenged WT and heterozygous mice both demonstrated significant AHR. By contrast, Muc5ac mice were protected (Fig. 2a; Supplementary Fig. 1b). To confirm these findings, we also assessed AHR in C57BL/6 and $\mathrm{BALB} / \mathrm{c}$ WT and congenic $M u c 5 a c^{-1-}$ mice exposed to aerosolized Aspergillus oryzae extract (AOE). AOE is a fungal allergen pertinent to human asthma that upon mucosal sensitization elicits strong asthma-like responses in mice ${ }^{38}$. In WT animals, AOE challenge caused $\mathrm{AHR}$ to $\mathrm{MCh}$, but in both $M u c 5 a c^{-1-}$ lines, AHR was abolished (Fig. 2b,c; Supplementary Fig. 1b). Importantly, protection occurred irrespective of C57BL/6 and BALB/c strain-specific airway- and tissue-localized 
a

b

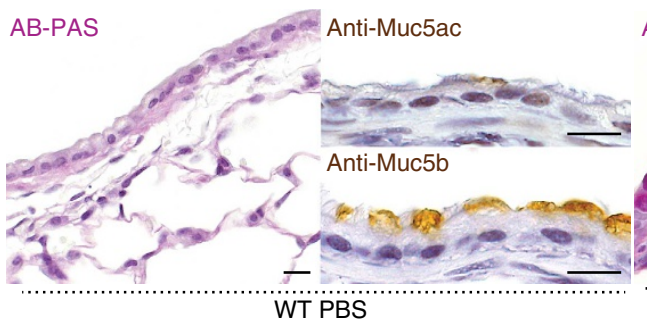

AB-PAS

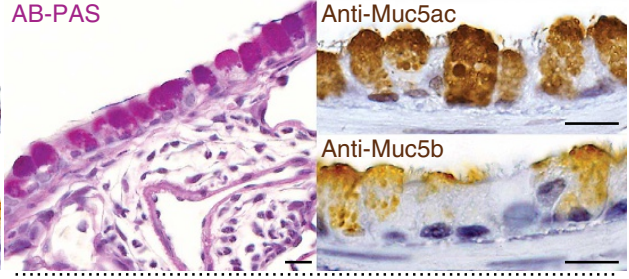

WT OVA

C

d

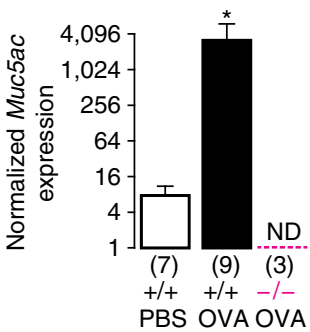

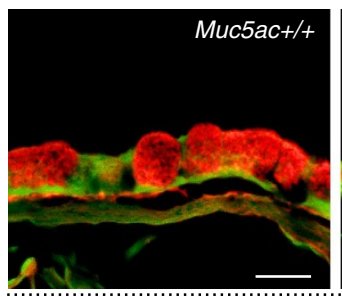

OVA e

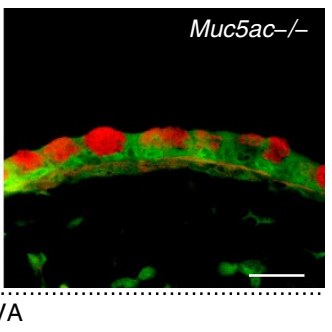

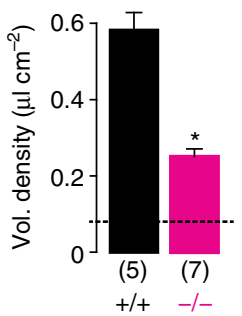

Figure 1 | Mucous metaplasia is significantly reduced in Muc5ac $^{-/-}$airways. (a and b) Mucin production in saline- (a) and OVA- (b) challenged WT mice. AB-PAS, anti-Muc5ac and anti-Muc5b staining shows upregulated Muc5ac and sustained Muc5b in allergic airways. (c-e) Muc5ac messenger RNA increased 383-fold in OVA-challenged WT mice $(+/+)$, but it was undetectable in Muc5ac knockout $(-/-)$ mice (c), resulting in a 67\% reduction in mucous metaplasia detected by periodic acid fluorescent Schiff's staining (d and e). Scale bars, $20 \mu \mathrm{m}$ (a,b,d). Mean, s.e.m. (c and e). ${ }^{\star} P<0.05$ (one-tailed) by a unpaired $t$-test (c and $\mathbf{e}$ ), with Welch's correction for unequal variances in $\mathbf{c}$. ND, not detected. Numbers in parentheses, ' $N$ ' mice. 'Vol.', volume. Dashed line in e, baseline in saline-challenged WT mice.
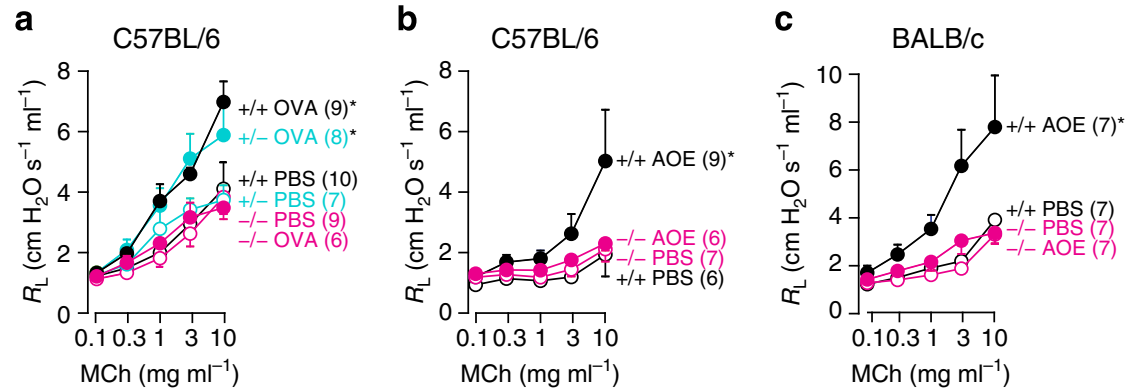

Figure 2 | Muc5ac is required for AHR to MCh. Lung resistance $\left(R_{\mathrm{L}}\right)$ responses to MCh in WT $\left(+/+\right.$, black), Muc5ac ${ }^{+/-}$(turquoise) and Muc5ac ${ }^{-/-}$ (magenta) mice after saline (open circles), OVA (closed circles in a) or Aspergillus oryzae extract (AOE; closed circles in b and c). Mean, s.e.m.

$(\mathbf{a}-\mathbf{c}) .{ }^{\star} P<0.05$ between slopes of best-fit regression lines by one-way ANOVA. Numbers in parentheses, ' $N$ ' mice.

pathophysiologies. To partition AHR on airway and tissue scales, we assessed lung mechanics by measuring input impedance. As reported previously ${ }^{39}$, AHR was strongly influenced by tissue resistance and elastance in C57BL/6 mice (Fig. 3a; Supplementary Fig. 2) and by tissue and central airway resistance in BALB/c mice (Fig. 3b; Supplementary Fig. 2); Muc5ac deficiency was protective irrespective of the background strain. Thus, in diverse models of allergic asthma, Muc5ac was required for AHR to MCh.

Mucin secretion alone is not sufficient to cause AHR. Because acute mucin secretion was required for AHR to MCh with no residual effect attributable to ASM hypercontractility (Figs 2 and 3), we next determined whether mucin secretion was sufficient to acutely induce AHR independently of bronchoconstriction. In airway epithelial cells, $\mathrm{P}_{2} \mathrm{Y}_{2}$ purinergic receptor stimulation is a major pathway for mucin secretion ${ }^{40} \cdot \mathrm{P}_{2} \mathrm{Y}_{2}$ receptors are activated by the endogenous agonists ATP and uridine triphosphate trisodium salt (UTP) ${ }^{40,4 P}$. ATP is rapidly dephosphorylated to adenosine, a signalling molecule that itself induces bronchoconstriction; ATP was thus inappropriate for these studies. Since UTP and its metabolites do not cause acute ASM contraction ${ }^{42}$, we challenged WT C57BL/6 mice with OVA or saline, and $72 \mathrm{~h}$ later we mechanically ventilated them and measured immediate changes in secretion and lung resistance in response to UTP. In OVA-challenged WT mice, although UTP rapidly stimulated secretion (Fig. 4a,b), there was no disruption of airflow (Fig. 4c). Thus, although Muc5ac was critical for allergic AHR in the presence of ASM-mediated bronchoconstriction following MCh, UTP-induced mucin secretion alone did not evoke an AHR response.

Allergic inflammation fails to drive AHR in Muc5ac null mice. Type-2 inflammation is critical for AHR in allergic mouse models ${ }^{30,31}$. Given the essential role for Muc5ac in AHR identified here (Figs 2 and 3), and the requirement for Muc5b in inflammatory cell clearance ${ }^{25}$, we determined whether Muc5ac expression mediated AHR by modulating allergic inflammation. 


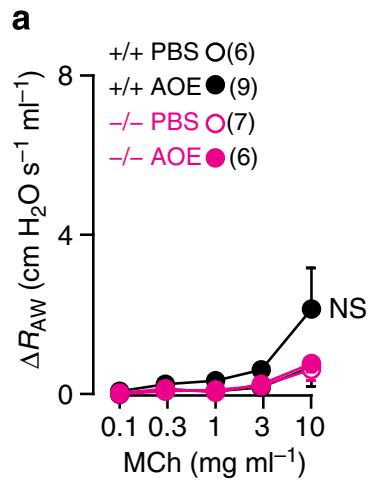

b

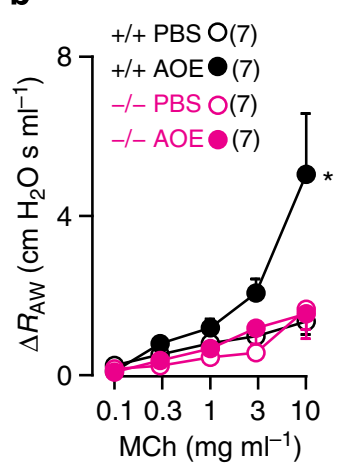

C57BL/6

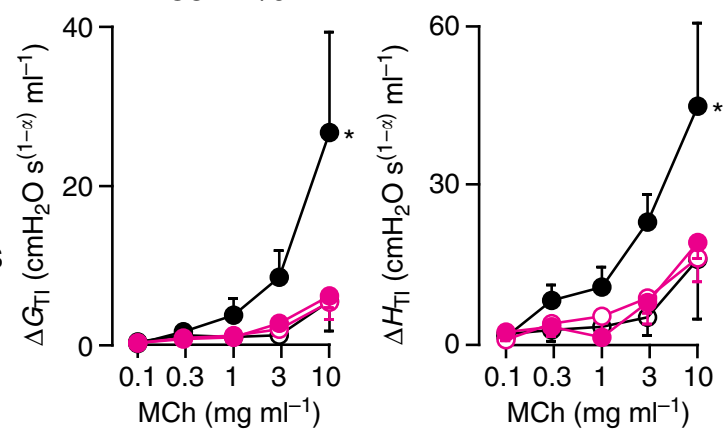

$\mathrm{BALB} / \mathrm{c}$

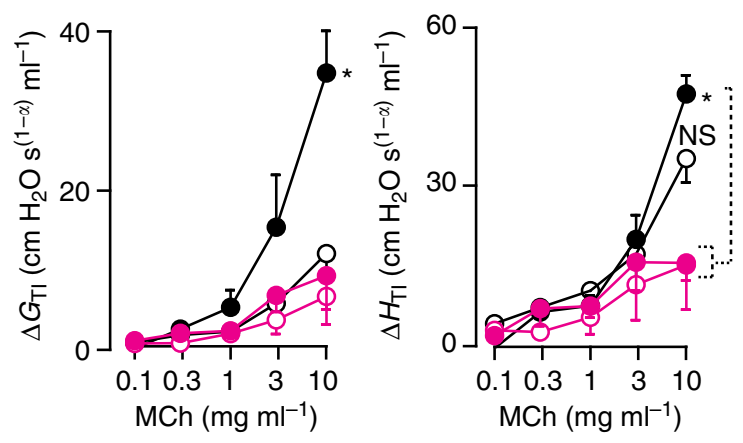

Figure 3 | Muc5ac mediates mouse strain-specific allergic airway pathophysiology. Conducting airway resistance $\left(\Delta R_{\mathrm{AW}}\right)$, tissue resistance $\left(\Delta G_{\mathrm{T}}\right)$ and tissue elastance $\left(\Delta H_{\mathrm{TI}}\right)$ were measured using oscillatory mechanics to assess input impedance. (a) In WT C57BL/6 mice, Aspergillus oryzae extract (AOE)-induced AHR (closed black circles) was dominated by changes in peripheral tissue mechanics $\left(\Delta G_{\mathrm{TI}}\right.$ and $\left.\Delta H_{\mathrm{TI}}\right)$ compared with changes in $R_{\mathrm{AW}}$. (b) In WT BALB/c mice, AOE-induced AHR (closed black circles) had central airway $\left(\Delta R_{\mathrm{AW}}\right)$ and tissue dominance $\left(\Delta G_{\mathrm{TI}}\right)$, whereas $\Delta H_{\mathrm{TI}}$ was not significantly different following AOE (NS) in WT mice. In both congenic strains, responses were significantly blunted in $\mathrm{Muc5}^{-\mathrm{c}^{-} /-}$mice (closed magenta circles). Mean, s.e.m. ${ }^{\star} P<0.05$ between slopes of best-fit regression lines by one-way ANOVA. 'NS', not significant. Numbers in parentheses, ' $N$ ' mice.

Compared with saline-challenged mice, there were significant increases in leukocyte numbers that varied with environmental exposure (OVA versus AOE) and genetic diversity (C57BL/6 versus $\mathrm{BALB} / \mathrm{c}$ ) (Supplementary Table 1). In WT C57BL/6 mice, there was an 8.5-fold difference in eosinophilic responses to OVA and AOE; between WT BALB/c and C57BL/6 strains, there was a 9.7-fold difference in eosinophil numbers in response to $\mathrm{AOE}$ (Fig. 5). However, between WT and $\mathrm{Muc5}_{\mathrm{ac}} \mathrm{C}^{-1-}$ mice in each of these groups, eosinophilic inflammation was not significantly different (Fig. 5; Supplementary Fig. 3; Supplementary Table 1). Thus, full protection from AHR in the absence of Muc5ac occurred although asthma-like allergic inflammation was sustained.

Reduced heterogeneous airway closure in Muc5ac null mice. Since AHR was abolished in $M u c 5 a c^{-1-}$ mice although inflammation remained intact, we hypothesized that AHR was mediated by physical obstruction of the airway lumen by Muc5ac. Obstruction could result from uniformly thickened mucus causing an increase in resistance to airflow via homogeneous changes in mean airway lumen diameter. Alternatively, obstruction could result from non-uniform mucous aggregates causing an increase in resistance to airflow via heterogeneous occlusion and airway closure (Fig. 6a). To compare these, AOE-challenged WT and $M u c 5 a c^{-/-}$mice were mechanically ventilated and treated with increasing doses of MCh as shown in Fig. 2. During peak changes in resistance following $10 \mathrm{mg} \mathrm{ml}^{-1} \mathrm{MCh}$, lungs were fixed transmurally with methacarn to preserve airway geometry and enable quantification of secreted mucus in situ ${ }^{43}$.
We generated a model of homogenous obstruction by fitting changes in resistance and airway lumen diameter to Poiseuille's law. In AOE-challenged $M u c 5 a c^{-1-}$ mice, resistance $(R)$ to airflow decreased 2.3-fold (Fig. 6b). Assuming laminar flow, Poiseuille's law predicts a $23 \%$ increase in mean lumen diameter uniformly (Fig. 6c, magenta dashed line). However, our histological measurements showed that mean lumen diameters improved by $39 \%$ and $32 \%$ in bronchi and bronchioles, respectively (Fig. 6d). If applied to the model above, airflow resistances would be three- to fourfold improved (Fig. 6c, black lines), but they were not. Thus, a model in which the prevention of AHR in $M u c 5 a c^{-/-}$mice was the result of protection from uniform mucus thickening and homogenous airflow obstruction is incongruent with our observations. Indeed, the poor fit of the findings above, the anatomically diverse responses observed in our AHR studies (Fig. 3a,b) and previous data from murine and computational models ${ }^{2,8}$ more strongly support heterogeneous obstruction as the major determinant of AHR.

To determine whether Muc5ac-mediated heterogeneous obstruction contributes to AHR, we analysed the distributions of individual airway lumen sizes relative to their anatomical locations. We observed non-uniform distributions of lumen sizes. In $M u c 5 a c^{-1-}$ lungs, there was a rightward shift in the distribution of airway lumen sizes compared with WT (Fig. 6e,f), which was consistent with protection from AHR. Furthermore, in WT mice, we also observed markedly greater proportions of bronchiolar airways with diameters of $\leq 100 \mu \mathrm{m}$ (Fig. 6f), a measurement consistent with a previously calculated threshold for airway closure ${ }^{8}$. Because components such as the epithelial and ASM layers and secreted mucus can all influence 
a
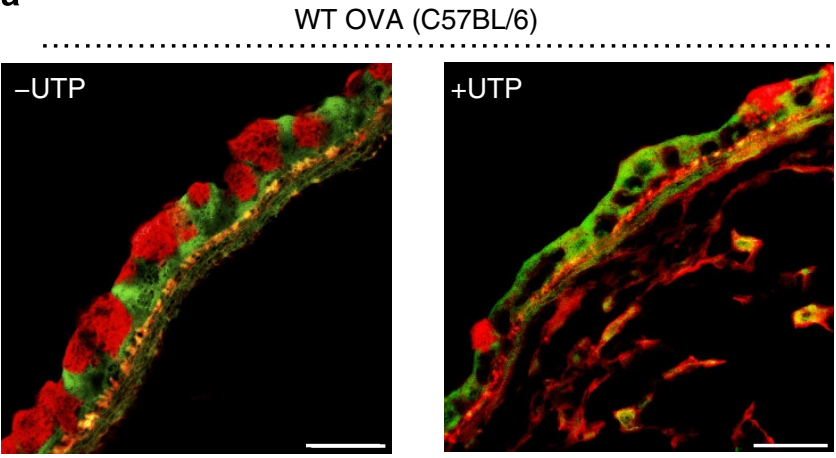

b

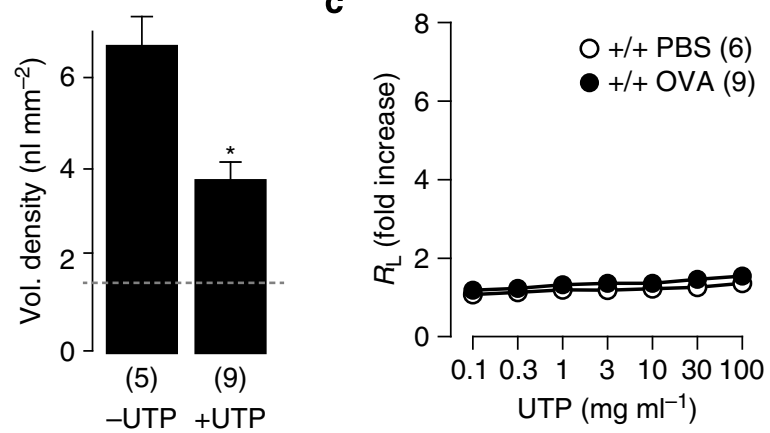

Figure 4 | Acute mucin secretion does not cause AHR. WT mice were OVA or PBS challenged, and then anaesthetized, ventilated and exposed to UTP (0.1-100 $\mathrm{mg} \mathrm{ml}^{-1}$ ) or vehicle (saline). (a) In OVA-challenged lungs fixed immediately after dose-response tests, there was an acute decrease in periodic acid fluorescent Schiff's (PAFS) staining in UTP (right panel) compared with vehicle-(' - UTP', left panel) treated mice, demonstrating acute UTP-induced secretion. Scale bars, $20 \mu \mathrm{m}$. (b) Quantitation of UTPinduced changes in PAFS staining in OVA-challenged mice. (c) No effect of UTP on $R_{\mathrm{L}}$ in either PBS- or OVA-challenged WT mice. Mean, s.e.m. in $\mathbf{b}$ and c. Numbers in parentheses, ' $N$ ' mice. 'Vol.', volume. Dashed line in $\mathbf{b}$, baseline in saline-challenged WT mice. ${ }^{\star} P<0.05$ by the $t$-test.

airway closure, we next determined how these were affected in $\mathrm{Mu} 5 \mathrm{ac}-1-$ mice.

In our functional studies, epithelial mucin production/secretion and ASM contraction were both required for AHR as shown in Figs 2-4. We thus compared the specific contributions of secreted mucin, epithelial thickening and ASM to anatomically localized changes in airway sizes. In bronchi, overall airway diameter (including ASM, epithelium, mucus and lumen) was $539 \pm 78 \mu \mathrm{m}$ in WT mice and $683 \pm 31 \mu \mathrm{m}$ in $M u c 5 a c^{-1-}$ mice ( $P=0.051$, one-tailed $t$-test), and in the bronchioles, these values were $274 \pm 15 \mu \mathrm{m}$ and $310 \pm 22 \mu \mathrm{m}$, respectively $(P=0.096$, onetailed $t$-test). Although not statistically significant as a combined group, these data suggested that secreted Muc5ac in the airway lumen could have some effect(s) on the behaviour of underlying tissues. We therefore derived a measure of composite airway wall/ mucus thickness by subtracting airway lumen diameters (Fig. 6d) from overall airway diameters. In the bronchi, this was not significantly different between WT and $M u c 5 a c^{-1-}$ mice $(103 \pm 21$ versus $76 \pm 9 \mu \mathrm{m}$, respectively; $P=0.124$, one-tailed $t$-test). By contrast, in the bronchioles, differences in composite airway wall/mucus thickness were significant $(71 \pm 14 \mu \mathrm{m}$ in WT versus $44 \pm 10 \mu \mathrm{m}$ in $M_{u c 5 a c^{-P}}$; $P=0.006$, one-tailed $t$-test). Mucus contribution to composite airway wall/mucus thickness was reduced in $M u c 5 a c^{-1-}$ mice compared with WT as expected, since a major mucin component was missing (Fig. 6g). However, there were also significant differences in

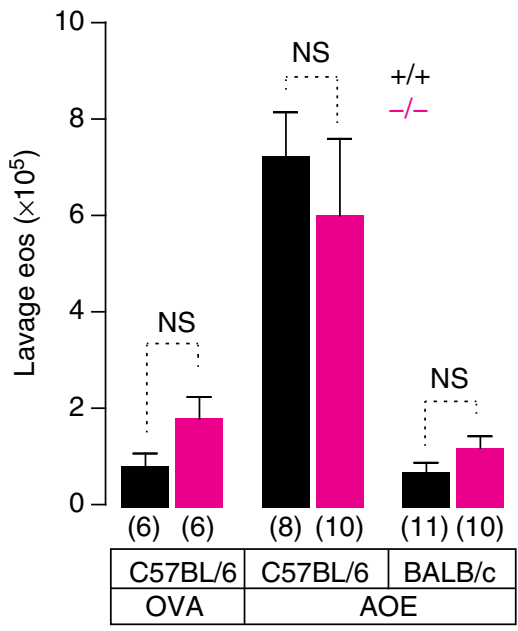

Figure 5 | Muc5ac deficiency does not reduce allergic inflammation. WT (black) and $\mathrm{Muc5ac}^{-/-}$(magenta) mice had similar numbers of eosinophils in lung lavage fluid irrespective of OVA or Aspergillus oryzae extract $(A O E)$ antigen challenge or strain background variables. For all groups, saline-challenged mice had $<10^{4}$ eosinophils in lung lavage fluid (see Supplementary Table 1). Mean, s.e.m. $N=6-11$ mice per group.

epithelial tissue and ASM components (Fig. 6h,i). Epithelial hypertrophy is directly related to mucin production ${ }^{9}$, so its contribution was also predictable (Fig. 6h). By contrast, the effect of Muc5ac deficiency on the contribution of ASM, which was significantly lower in $M u c 5 a c^{-1-}$ bronchi and bronchioles, was surprising (Fig. 6i).

Collectively, these findings reinforce the concept AHR is caused by contractile and non-contractile factors that contribute interdependently. Since AHR in allergic mouse lungs was dependent on a combination of ASM contraction and Muc5ac secretion (Figs 2-4), and since changes in airway structure and function did not fit models of homogenous changes in airflow, these findings further support the hypothesis that epithelial Muc5ac secretion during MCh-induced ASM constriction causes the formation of mucous plugs that potentiate heterogeneous airflow disruption.

Muc5ac mediates heterogeneous mucous occlusion in mice. In WT airways, mucous plugs were present and Muc5ac was abundant (Fig. 7a); in $M u c 5 a c^{-1-}$ airways, plugs were infrequent and mucus was sparse (Fig. 7b). Throughout the lungs, mean lumen mucin volume density, a homogeneous measure of mucous content across airway surfaces, was reduced by $32 \%$ (Table 1). Thus, as with lumen diameter, assuming uniform mucus accumulation across all airway surfaces and fitting these to Poiseuille's law results in changes in resistance that exceed those observed experimentally.

We thus determined the extent and distribution of mucous occlusion percentage, a sensitive measure of the fractional volume of airspaces occupied by mucus. This was used previously to quantify the prevalent but heterogeneous airway plugging observed in fatal asthma, which showed that $>98 \%$ of airways in these patients were occluded with mucus by $20 \%$ or more ${ }^{15}$. In Muc5ac $-/-$ airways, overall mean lumen occlusion percentage was reduced by $\sim 60 \%$ (Table 1 ; Fig. 7c). Similar to human asthma ${ }^{15}$, plugging was also heterogeneous in mice (Fig. 7d). In WT airways, median occlusion was $6.7 \%$ compared with $0 \%$ in $M u c 5 a c^{-/}$- airways. Furthermore, in WT mice only $12 \%$ of airways were occlusion 
a
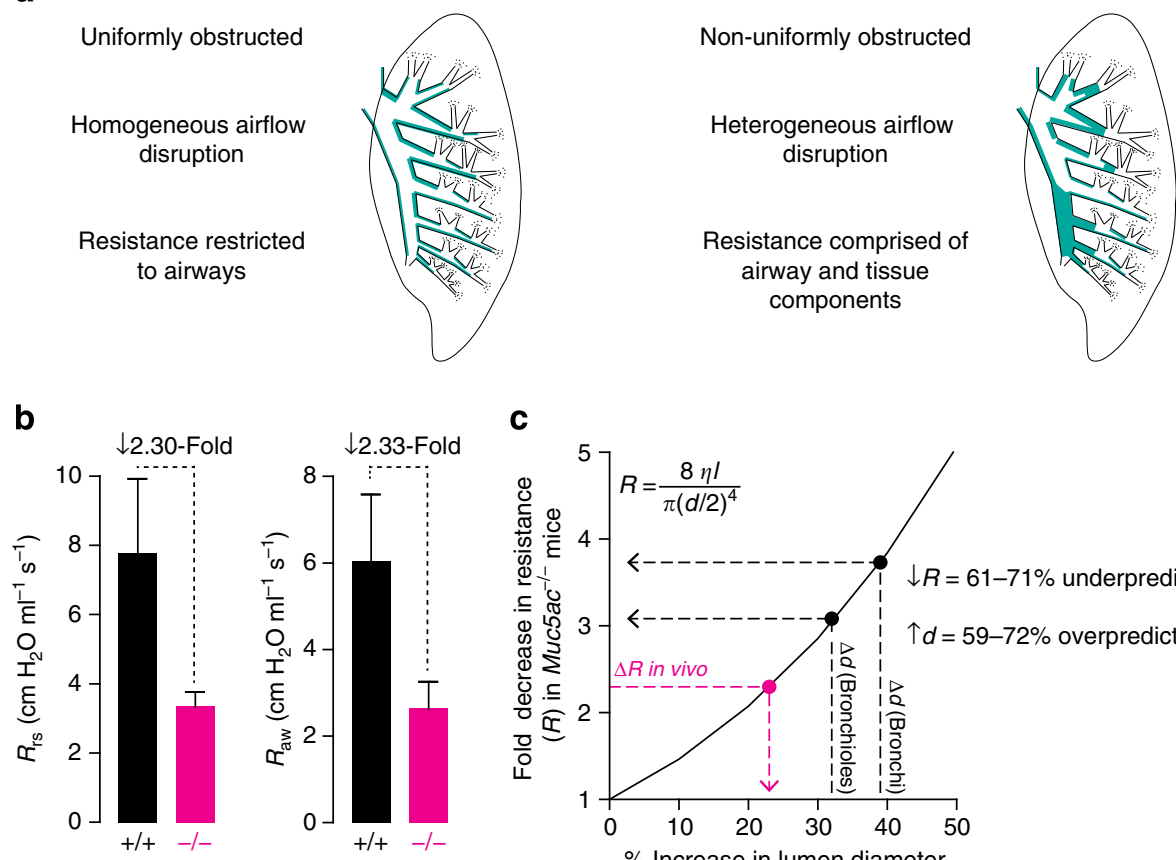

C
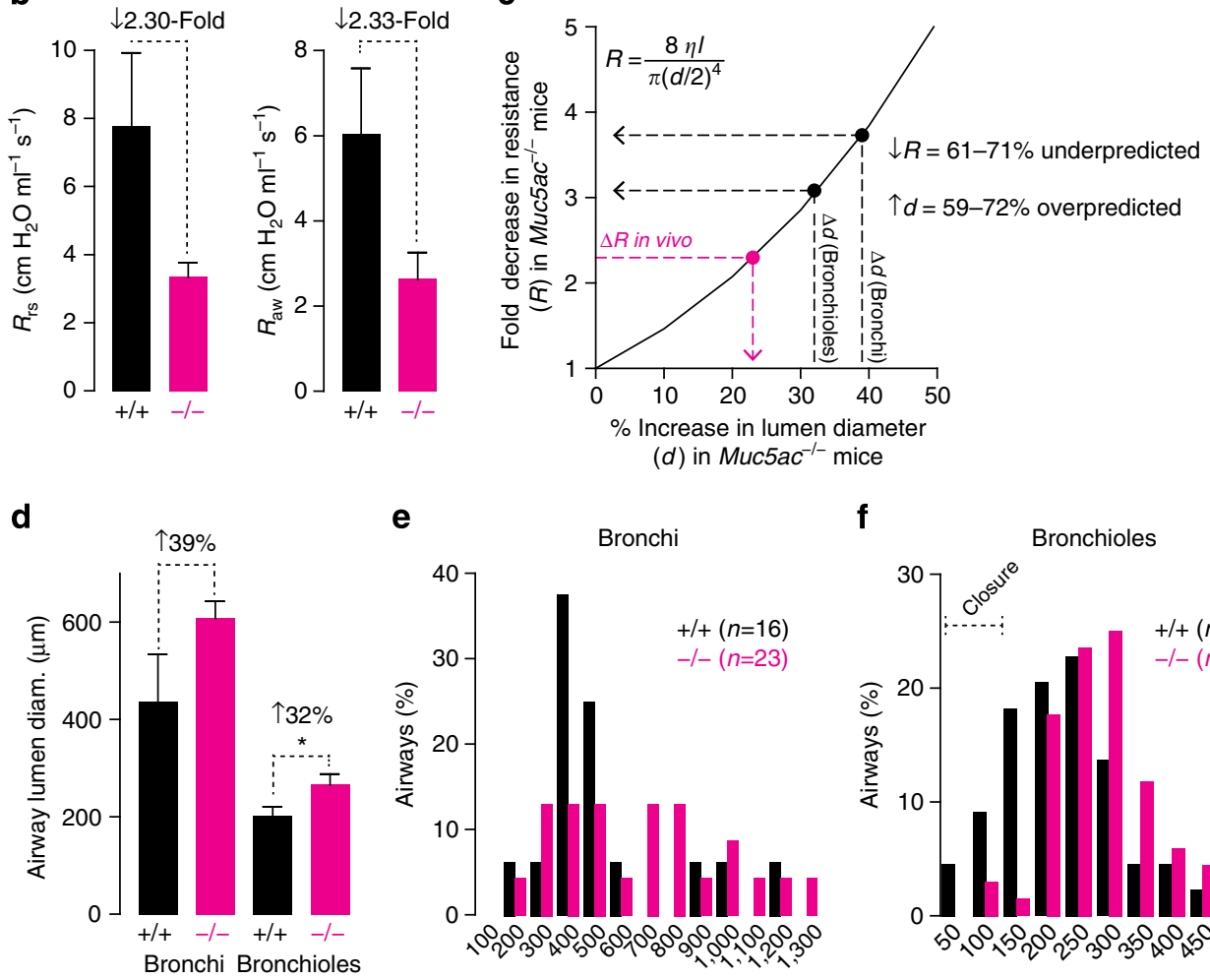

$\mathbf{e}$
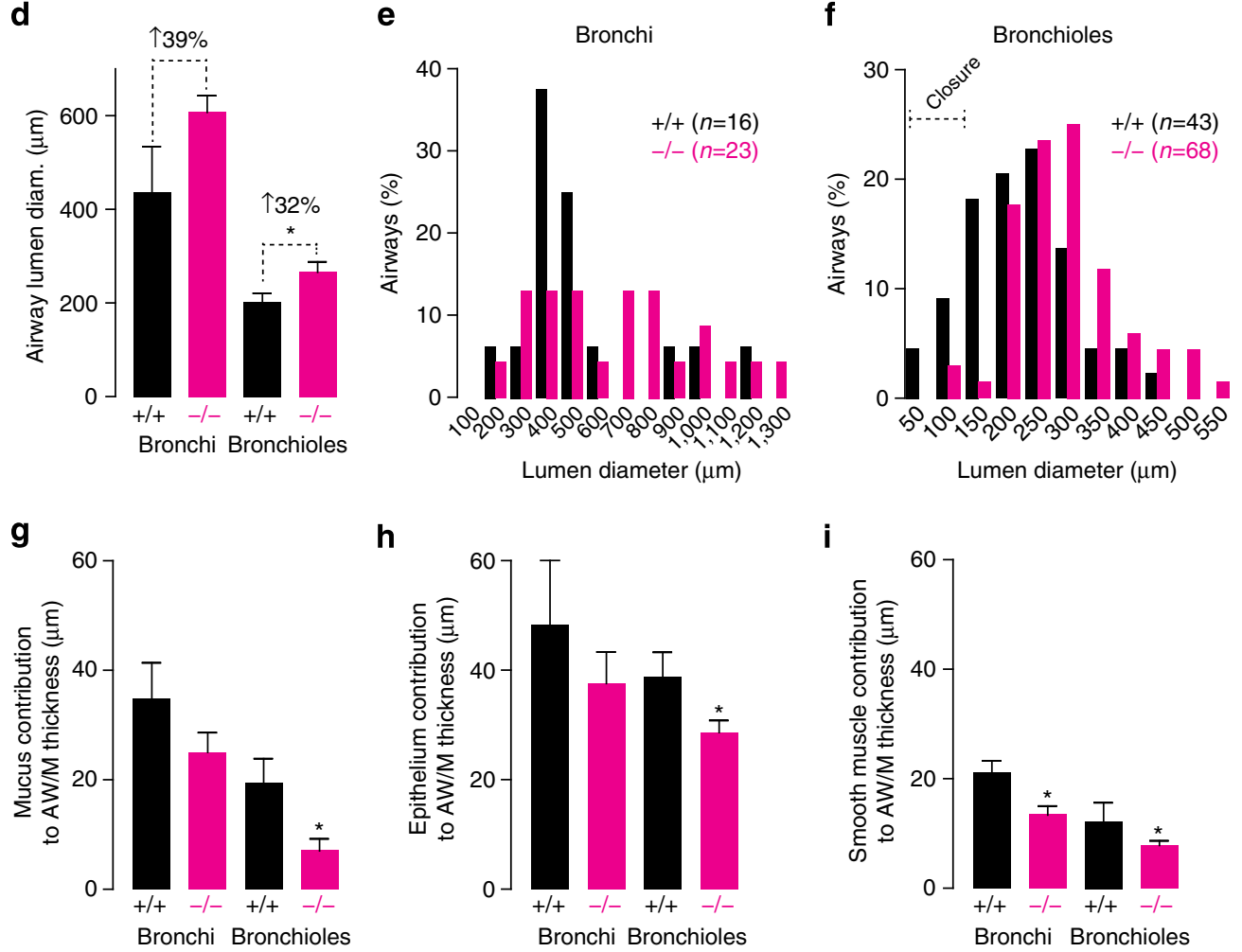

$\mathbf{h}$

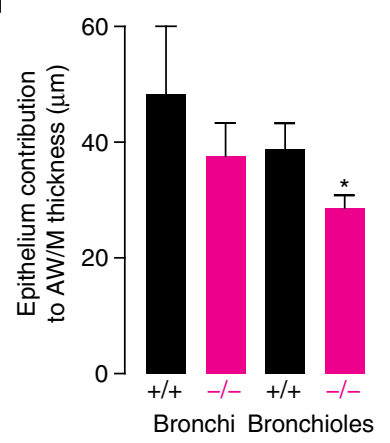

i

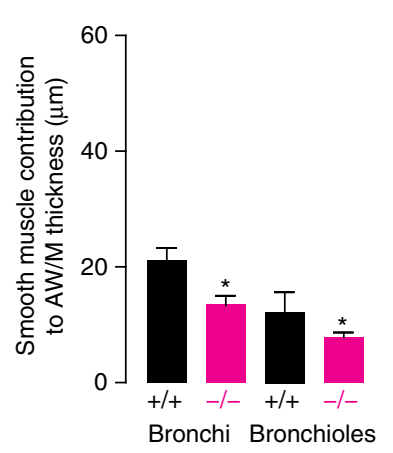

Figure 6 | Muc5ac deficiency protects against heterogeneous airflow obstruction. (a) Models of the effects of uniform versus non-uniform obstruction on airflow. (b and $\mathbf{c}$ ) Changes in peak total lung and airway resistances $(\Delta R)$ following $10 \mathrm{mg} \mathrm{ml}^{-1} \mathrm{MCh}$ in WT and Muc5ac ${ }^{-/-}$mice $(n=7$ per genotype) (b) were fit to Poiseuille's law (equation in c) to predict changes in airway lumen diameter (magenta point and dashed arrow). Viscosity ( $\eta$ ) and length $(I)$ were held constant. (d-f) Mean $(\mathbf{d})$ and distributions $(\mathbf{e}, \mathbf{f})$ of airway lumen diameters measured in methacarn-fixed lungs; changes in mean lumen diameter were also fit to the equation in $\mathbf{c}$ (black points and dashed arrow). (g-i) Contributions of mucus, airway epithelium and smooth muscle to changes in composite airway wall/mucus (AW/M) thickness. Mean, s.e.m. in b,d and $\mathbf{g}$-i. $x$ axis labels bin maximum values in $\mathbf{e}$ and $\mathbf{f}$. $N=4$ WT and 5 $\mathrm{Muc5ac}^{-/-}$mice. ${ }^{\star} P<0.05$ by a $t$-test.

free, and $32 \%$ were occluded by $\geq 20 \%$. In $\mathrm{Muc5}_{\mathrm{ac}} \mathrm{C}^{-/-}$mice, $51 \%$ of airways were occlusion free, and only $8.2 \%$ were occluded by $\geq 20 \%$.
To further dissect the sources of heterogeneous obstruction, we determined the distribution of occlusions by airway generation. In WT mice, occlusions of $\geq 20 \%$ were common in axial bronchi 
a

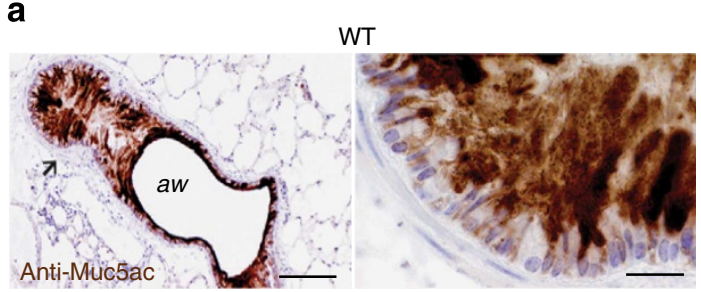

b

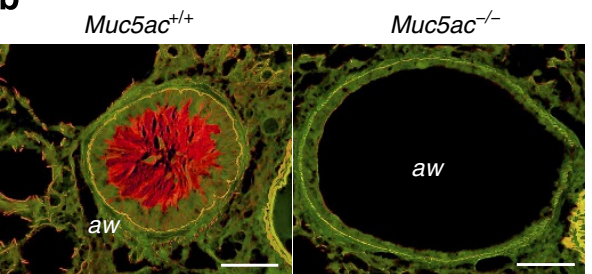

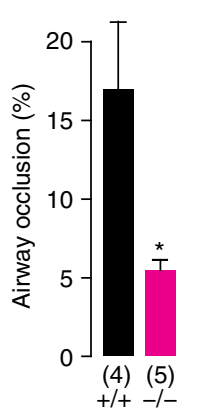

d

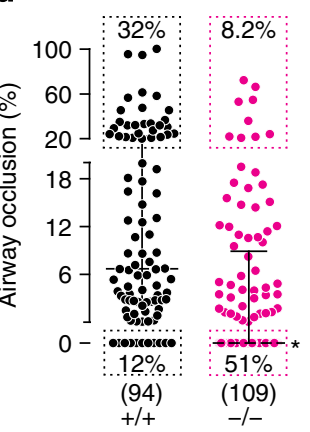

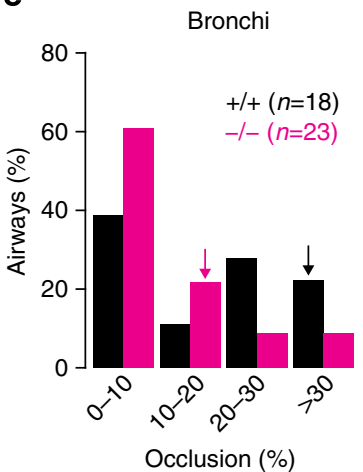

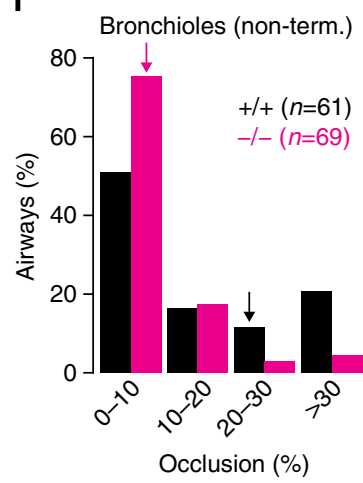

Figure 7 | Muc5ac deficiency reduces mucous plugging. (a and $\mathbf{b}$ ) Inhaled MCh caused mucous plugging in WT allergic mouse airways (aw). Muc5ac protein (a) and PAFS staining (b) in AOE-challenged WT $\left(\mathrm{Muc5ac}^{+/+}\right)$and $\mathrm{Muc5ac}^{-/-}$mouse airways. Arrow in a, region is shown at a higher magnification in the right panel. (c-f) Extent and distribution of mucous plugging. Mean occlusion per mouse across all measured airways (c). Distribution of the extent (d) and localization (e and $\mathbf{f}$ ) of occlusions. Arrows in $\mathbf{e}$ and $\mathbf{f}$, starts of upper quartiles. Scale bars, 100 and $20 \mu \mathrm{m}$ (a); $50 \mu \mathrm{m}$ (b). Mean, s.e.m. (c). Median quartiles (d). ${ }^{\star} P<0.05$ (one-tailed) by an unpaired $t$-test (c) and the Mann-Whitney test (d). Numbers in parentheses, ' $N$ ' mice (c), ' $N$ ' airways (d-f).

\section{Table 1 | Measurements of secreted mucus in mice during bronchoconstriction.}

\begin{tabular}{|c|c|c|c|}
\hline Histological measurement & WT & Muc5ac $-/-$ & $P$ value \\
\hline \multicolumn{4}{|c|}{$V_{\text {mucus }}$ per surface area $\left(\mathrm{mm}^{3} \mathrm{~cm}^{-2}\right)$} \\
\hline All airways & $0.71 \pm 0.08$ & $0.48 \pm 0.43$ & 0.313 \\
\hline Axial bronchi & $1.16 \pm 0.09$ & $0.84 \pm 0.11^{\star}$ & $0.032^{\star}$ \\
\hline Non-terminal bronchioles & $0.58 \pm 0.13$ & $0.30 \pm 0.06^{\star}$ & $0.041^{\star}$ \\
\hline \multicolumn{4}{|c|}{$V_{\text {mucus }}$ per anatomic region $\left(\mathrm{mm}^{3} \mathrm{~mm}^{-3}\right)$} \\
\hline All airways & $3.9 \pm 1.1$ & $2.3 \pm 0.6$ & 0.185 \\
\hline Axial bronchi & $0.8 \pm 0.1$ & $0.6 \pm 0.2$ & 0.122 \\
\hline Non-terminal bronchioles & $3.1 \pm 1.0$ & $1.7 \pm 0.5^{\star}$ & 0.099 \\
\hline \multicolumn{4}{|c|}{$\%$ Occlusion per anatomic region } \\
\hline All airways & $18 \pm 4.3$ & $7.2 \pm 1.4$ & $0.020^{\star}$ \\
\hline Axial bronchi & $20.1 \pm 5.7$ & $11.7 \pm 2.6$ & 0.097 \\
\hline Non-terminal bronchioles & $16.8 \pm 1.3$ & $6.3 \pm 1.2$ & $0.022^{*}$ \\
\hline
\end{tabular}

WT, wild type.

Values are means \pm s.e.m. ${ }^{\star} P<0.05$ by a unpaired $t$-test. $N=4 \mathrm{WT}$ and $5 \mathrm{Muc5ac}^{-/-}$mice.

(50\% of airways) and bronchioles (31\% of non-terminal airways) (Fig. 7e,f). In $M u c 5 a c^{-1-}$ mice, occlusions of $\geq 20 \%$ were present in only $17.4 \%$ of axial bronchi (2.7-fold reduction) and $7.2 \%$ of bronchioles (4.6-fold reduction). Thus, disruption of the $M u c 5 a c$ gene in mice resulted in protection from a heterogeneous airway plugging phenotype that was similar to the pathology observed in fatal human asthma ${ }^{15}$.

\section{Discussion}

AHR in asthma is currently thought to result primarily from inflammation-triggered ASM hypercontractility ${ }^{1}$. However, there is growing evidence that in addition to ASM-driven narrowing, obstruction is also mediated by significant non-contractile factors $2,4,8,44$. Here we report that in allergically inflamed mouse lungs, Muc5ac is an essential non-contractile mediator of AHR. These findings have important implications with respect to mechanisms of asthma pathophysiology. They could also inform the development of more effective treatments that target MUC5AC. These could especially benefit patients in whom bronchodilators or corticosteroids are ineffective ${ }^{1,45}$.

In our studies, mucin secretion and ASM contraction were both required for AHR. Although there were C57BL/6 and BALB/ $C$ strain-specific differences between central airway and peripheral tissue responses, there was no residual AHR to $\mathrm{MCh}$ in $M u c 5 a c^{-}-$mice at whole-lung or partitioned airway and tissue scales. Thus, our data challenge the notion that AHR in allergic asthma is dominated by exaggerated ASM contraction. That dogma is grounded on studies showing ASM hypertrophy in pathological tissues, hypercontractility of excised ASM from asthma patients and on protection provided by bronchodilating $\beta$-agonist treatments ${ }^{1}$. Such findings show association and suggest necessity for ASM hypercontractility in AHR, but they do not prove its sufficiency. Our studies also showed that inducing mucin secretion without bronchoconstriction did not evoke AHR. Thus, in mice, while ASM contraction and mucin secretion are both necessary for acute AHR, neither alone is sufficient. Rather, ASM contraction and mucin secretion mediate AHR cooperatively. These findings highlight the need to better understand coordinated contractile and non-contractile mechanisms of airflow obstruction in asthma ${ }^{2}$.

In addition to finding that ASM contraction and Muc5ac secretion were both required for AHR, we also found an apparent interdependence between mucus secretion and ASM-mediated airway constriction. Airway diameters and ASM thickness were both significantly reduced in allergic $M u c 5 a c^{-1-}$ lungs after MCh (Fig. 6). This may reflect effects of mucus secretion on airway surface tension and the propensity for airway closure, or it 
may demonstrate the ability of airways with less mucus to remain open following deep lung inflation manoeuvres that are administered between MCh doses in our experimental set-up. In asthma patients, these could affect the ability of deep breaths to maintain open airways. Alternatively, during dose-response tests in our studies, enhanced passage of aerosolized MCh through open airways in $M u c 5 a c^{-1-}$ mice could have increased its deposition in the terminal bronchioles, which have little to no smooth muscle or mucin expression and thus contribute minimally to AHR. Lastly, changes in ASM constriction could have been related to changes in ASM hypertrophy in allergic $M u c 5 a c^{-1-}$ mice. However, this is unlikely since the total amount of ASM was not different between allergic WT and $M u c 5 a c^{-1-}$ mice (Supplementary Table 2). Nonetheless, further investigation is needed to determine the potential significance and mechanisms of these intriguing interactions between ASM and non-contractile components.

To this end, other non-contractile factors of AHR have been studied $^{2,3,8}$. Plasma extravasation and fibrin accumulation can reduce airway liquid surface tension and cause acute airway closure $^{3}$. Studies in animal and computational models present this alternative to ASM dominance as heterogeneous closure occurring via 'sudden liquid bridge formation', ${ }^{2,8}$. Our findings are consistent with this conceptual framework, which we advance by defining Muc5ac as a key molecular mediator. Indeed, it is plausible that aqueous-soluble factors become enriched within hydrated and heterogeneously distributed Muc5accontaining mucous aggregates. Alternatively, each could occur independently yet synergize to mediate airway closure during bronchoconstriction.

Our studies also demonstrated that Muc5ac mediates AHR independently of the degrees of allergic inflammation. Differences in genetic and stimulus-dependent allergic inflammation were striking (Fig. 5; Supplementary Fig. 3; Supplementary Table 1). However, although inflammation is critical for allergic $\mathrm{AHR}^{30,31}$, and secreted mucins can modulate immune function and resolve inflammation $25,26,46$, eosinophils in OVA- and AOE-challenged Muc5ac-l- lungs did not differ from WT (Fig. 5). By contrast, mucous plugs throughout $M u c 5 a c^{-1-}$ airways were significantly reduced in abundance and severity (Fig. 7). Thus, protection from AHR was mediated not by altered inflammation but through reduced plug formation: in constricting airways, acute physical obstruction caused by Muc5ac was essential for AHR. This segregation between Muc5ac function and preserved type-2 inflammation is consistent with in vivo findings in nematodeinfected mice. 'Allergic' type- 2 inflammation stems from off-target anti-helminth responses. In nematode-infected $M u c 5 a c^{-9-}$ mice, although intestinal type-2 inflammation is sustained, animals do not effectively control infections in the absence of Muc5 $\mathrm{ac}^{37}$. Rather than affecting inflammation, the type-2 inflammation effector Muc5ac (but not the homeostatic intestinal mucin Muc2) directly impairs worm viability ${ }^{37}$. It remains to be determined whether the mechanisms by which Muc5ac selectively inhibits worm growth also promote mucus aggregation and plugging in the airways.

Indeed, biochemical/biophysical mechanisms by which secreted mucus occludes airways in asthma are poorly understood. Our current understanding of mucins in asthma is largely limited to anecdotal evidence of altered physical properties ${ }^{19}$, to associations drawn from pathological studies showing increased goblet cell numbers and mucin contents in biopsies from a wide range of disease severities ${ }^{4,47}$ and to pervasive airway plugging in autopsy specimens obtained from fatal cases ${ }^{15-17}$. Some studies do show that patients have persistently abnormal mucus and mucociliary clearance $4,11,19,20,23,48,49$. However, the obstructive consequences of mucin secretion in AHR and non-fatal exacerbations have remained unclear. This may be due in part to a lack of appreciation of interrelated ASM contractile and epithelial secretory processes. MCh induces both ASM contraction and epithelial mucin secretion ${ }^{21,22}$. In the airways, mucins become hydrated ${ }^{50}$, swelling to produce a thick gel that amplifies the obstructive effects of narrowing initiated by $\mathrm{ASM}^{2,4}$. Applying strategies that combine relaxing ASM with reducing mucin production and secretion and/or enhancing mucociliary clearance could improve disease treatment.

While potentially beneficial, clearance-enhancing mucolytics and expectorants have been disregarded as asthma treatments. At achievable doses, existing therapies such as hypertonic saline and $\mathrm{N}$-acetylcysteine show poor efficacy and tolerability, because the high concentrations that are needed to clear mucous plugs are irritating and cause bronchoconstriction ${ }^{4,51-53}$. For example, although $N$-acetylcysteine is the only Food and Drug Administration-approved reducing agent available as an inhaled adjunct mucolytic, clinical data do not support its use ${ }^{54}$, reflecting its low mucolytic activity at high mucin concentrations ${ }^{55,56}$ and at a neutral airway $\mathrm{pH}\left(7.14\right.$ in mice $\left.{ }^{57}\right)$. We speculate that improved inhaled mucolytic agents can reduce plugging and AHR in asthma. However, it will be critical for any strategy that reverses or prevents obstruction, to do so safely while also protecting mucous-mediated defence.

Muc5ac is one of two polymeric mucins that are the predominant glycoproteins in airway mucus. The other, Muc5b, is expressed abundantly at baseline and remains present following allergic inflammation in mice (Fig. 1a,b). Muc5b is critical for homeostatic defence. $M u c 5 b^{-1-}$, but not $M u c 5 a c^{-1-}$, mice have severely impaired mucociliary clearance functions, resulting in accumulated particles and bacteria in the airways, and ultimately in death due to uncontrolled infection ${ }^{25}$. In humans with asthma, as MUC5AC expression increases, MUC5B expression either remains stable or decreases markedly ${ }^{10,12}$, with divergent MUC5AC:MUC5B expression ratios associated with worse $A H R^{10}$. Thus, targeting MUC5AC, while preserving or enhancing MUC5B, should be considered for any improved mucolytic therapies.

Two possible means for controlling the effects of MUC5AC on AHR are through targeting gene expression mechanisms or the glycoprotein itself. Critical expression signals include IL-13 and epidermal growth factor receptor ligands ${ }^{34-36}$. A monoclonal anti-IL-13 antibody has shown promise in early human trials ${ }^{29}$, and a small-molecule inhibitor of MAPK13 was effective in allergic mice ${ }^{58}$. However, a recent trial of epidermal growth factor receptor blockade in chronic obstructive pulmonary disease (COPD) failed, because at doses that achieved minimal changes in mucin production, adverse effects occurred ${ }^{59}$. Transcriptional control of MUC5AC/Muc5ac gene expression through regulators including SPDEF (SAM pointed domain-containing Ets transcription factor), Notch and hypoxia-inducible factor-1 (refs 24,60-62) have also been identified, but they are not currently targetable.

As an alternative to controlling gene expression, more selective results could potentially be yielded by disrupting the secreted MUC5AC polymer or by blocking its assembly. Recent studies have revealed mechanisms for controlling mucin disulfide bondmediated polymerization ${ }^{63}$ and patterns of selective mucin glycosylation ${ }^{23,49}$. These may provide better targets for preventing mucus aggregation and airway plugging acutely. Specifically, by determining which glycan or oligomerization mechanisms are specific for MUC5AC versus MUC5B, novel strategies could be designed to reduce AHR by selectively inhibiting MUC5AC-mediated plugging while maintaining MUC5B-mediated airway defence. Future studies are directed at investigating these possible mechanisms. Since excessive 
MUC5AC is also a common problem in airway diseases such as COPD and cystic fibrosis, whose inflammation and MUC5AC gene induction pathways differ significantly from allergic asthma, targeting the physical glycoprotein and polymeric properties of MUC5AC could have broader therapeutic applications.

\section{Methods}

Experimental animals. Studies were performed with approval of the animal care and use committees of the University of Colorado (approval number 97412(01)E) and the MD Anderson Cancer Center (approval numbers 05-04-046, 05-05-04334 and 00001214-RN00). $\mathrm{Muc5ac}^{-1-}$ mice were generated previously ${ }^{37}$. Mice were backcrossed onto congenic $\mathrm{C} 57 \mathrm{BL} / 6$ and $\mathrm{BALB} / \mathrm{c}$ lines using marker-assisted analysis at the MD Anderson Research Animal Support Facility-Smithville, Animal Genetics Services lab. After achieving 100\% carriage of 77 C57BL/6 and 82 BALB/c strain-specific markers, lines were backcrossed 3-5 additional generations. Mice were either bred as homozygous nulls or heterozygotes. $\mathrm{C} 57 \mathrm{BL} / 6$ and $\mathrm{BALB} / \mathrm{c}$ mice from the Jackson Labs (Bar Harbor, ME) were used for congenic backcrossing and as WT controls. Male and female mice were used starting at 7-8 weeks age. Before and after exposures, mice were housed under specific pathogen-free conditions in ventilated cages ( $\leq 5$ mice per cage) with a 12-h light/dark cycle and with food and water available ad libitum. Mice were placed into experimental and control groups chronologically.

Ovalbumin- and $\boldsymbol{A}$. oryzae induced lung inflammation. For studies in OVAchallenged mice, animals were sensitized intraperitoneally (i.p.) with 4 weekly injections of $20 \mu \mathrm{g}$ OVA, $2.25 \mathrm{mg}$ alum in saline (Sigma, St Louis, MO). Seven days after the last injections, sensitized mice were then exposed to $5 \mathrm{ml}$ of a $2.5 \%(\mathrm{w} / \mathrm{v})$ OVA aerosol delivered until the full volume was administered ${ }^{9}$. For AOE challenge, mice were exposed by the nose-only inhalational challenge. AOE is a commercially available fungal protease/peptidase complex produced by submerged fermentation of A. oryzae (catalogue no. P6110 Sigma). Although marketed as a 'protease,' our own microscopic analysis shows that it is a crude extract rich in fungal particles, although no live organisms were detected when samples were cultured. In addition, endotoxin levels were $<50$ units $\mathrm{ml}^{-1}$ (Endosafe-PTS, Charles River Laboratories, Wilmington, MA). AOE was used previously to elicit allergic inflammation in mice following bolus intranasal application ${ }^{38}$. For aerosol exposures here, a 5-ml volume of a $10 \% \mathrm{v} / \mathrm{v}$ solution of AOE was made in PBS containing $2.5 \% \mathrm{w} / \mathrm{v}$ OVA (Sigma) and $1 \% \mathrm{v} / \mathrm{v}$ antifoam A (Sigma). Aerosols were generated using an Ultravent jet nebulizer, which when driven at $30 \mathrm{psi}$, generates particles with a mean mass aerodynamic diameter of $<1 \mu \mathrm{m}^{64}$. In mice, $>80 \%$ of the particles generated are respirable and deposit in the lower airways efficiently ${ }^{64}$. Exposures were made in custom-built nose-only chambers and lasted until a 5-ml volume was delivered (40-45 min). Mice were AOE challenged once weekly for 4 weeks. Allergic inflammation was confirmed by lung lavage and Giemsa staining. Muc5ac messenger RNA levels were determined by quantitative PCR as described previously ${ }^{24}$

Airway hyperreactivity. Lung function was measured $72 \mathrm{~h}$ post OVA challenge or $48 \mathrm{~h}$ post AOE challenge using a flexiVent (Scireq, Montreal, Quebec, Canada). Mice were anaesthetized with $2.0 \mathrm{~g} \mathrm{~kg}^{-1}$ urethane (i.p.). This was sufficient to provide up to $4 \mathrm{~h}$ of sedation, although experiments lasted only $20-40 \mathrm{~min}$. Mice were tracheostomized with a blunt beveled $18 \mathrm{G}$ Luer stub adapter. Once ventilated (150 breaths per min, $10 \mathrm{ml} \mathrm{kg}^{-1}$, against $3 \mathrm{~cm} \mathrm{H}_{2} \mathrm{O}$ positive end expiratory pressure), mice were paralysed by continuous infusion of succinylcholine chloride $\left(10 \mu \mathrm{g} \mathrm{g}^{-1} \mathrm{~min}^{-1}\right.$, i.p.). Baseline total lung resistance $\left(R_{\mathrm{L}}\right)$ using a single-compartment model and the input impedance parameters, Newtonian (airway) resistance $\left(R_{\mathrm{AW}}\right)$, tissue resistance $\left(G_{\mathrm{TI}}\right)$ and tissue elastance $\left(H_{\mathrm{TI}}\right)$ were determined. Changes in $R_{\mathrm{L}}, R_{\mathrm{AW}}, G_{\mathrm{TI}}$ and $H_{\mathrm{TI}}$ in response to successive, increasing doses of MCh chloride (Sigma, Cat no. A2251; 0.1-10 $\mathrm{mg} \mathrm{ml}^{-1}$ ) or UTP (Sigma, Cat no 94370; 0.1-100 $\mathrm{mg} \mathrm{ml}^{-1}$ ) administered by an in-line ultrasonic nebulizer were assessed. Heart rates were monitored by electrocardiogram. At $10 \mathrm{mg} \mathrm{ml}^{-1} \mathrm{MCh}$, heart rates often fell below 200 beats per min and did not recover. At this point, no further dose-response measurements were made.

Mucin production and secretion. To assess mucin production, mouse lungs were fixed by intratracheal formalin administration and stained using periodic acid fluorescent Schiff s (PAFS) to assess and quantify intracellular glycoconjugates ${ }^{9,65}$. To assess secretion, WT C57BL/6 mice were OVA challenged, ventilated on a flexiVent and exposed to inhaled UTP to induce secretion $\left(0.1-100 \mathrm{mg} \mathrm{ml}^{-1}\right)$ or inhaled saline to control for ventilator- or vehicle-induced secretion (seven doses repeated over the same intervals used for UTP). After UTP or saline exposures, lungs were immediately fixed intratracheally with formalin. Acute mucin secretion in airway tissues was assessed by measuring changes retained in PAFS-positive contents. Secretion was defined as decreased staining due to acute depletion of intracellular stores in UTP versus saline-treated animals.
Mucous plugging and airway measurements. Tissues were fixed by adapting a method previously used to preserve colonic mucus using methanol-based Carnoy's fixative (methacarn $)^{43}$. Methacarn is a highly permeant non-crosslinking preservative that rapidly penetrates tissues transmurally while minimizing mucus disruption and tissue shrinkage ${ }^{43}$. Animals were carried through full dose-response curves. During peak increases in $R_{\mathrm{L}}$ following $10 \mathrm{mg} \mathrm{ml}^{-1} \mathrm{MCh}$, mice were rapidly euthanized by anaesthetic overdose and cessation of mechanical ventilation. Mice remained connected to the ventilator, which by default stopped at end expiration ( $3 \mathrm{~cm} \mathrm{H}_{2} \mathrm{O}$ positive end expiratory pressure). Skin covering the lower sternum was removed, and $400 \mu \mathrm{l}$ of methacarn was injected into the pleural space. The trachea was ligated immediately below the tracheal cannula. These steps took less than $15 \mathrm{~s}$, thus minimizing changes in airway constriction and mucus transport. Lungs were fixed in situ for 30-60 min, then removed and immersion fixed with the trachea still ligated for $24-48 \mathrm{~h}$ in methacarn at $4{ }^{\circ} \mathrm{C}$. Tissues were transferred to $100 \%$ methanol (changed twice over a 48 -h period). While remaining fully immersed in methanol, left lungs were sectioned into uniform 1.92-mm-thick filets and imaged to calculate volume by Cavalieri estimation $\left(319 \pm 31\right.$ and $322 \pm 24 \mathrm{~mm}^{3}$ for WT and $M u c 5 a c^{-1-}$ mice, respectively). Tissues were then paraffin processed and sectioned ( $5 \mu \mathrm{m}$ thickness)

Histological assessments were made using alcian blue-periodic acid Schiff's (AB-PAS) and periodic acid fluorescent Schiffs (PAFS) to assess total mucin, Muc5ac (clone 45M1, 1:100 dilution), and MUC5B (custom polyclonal antisera, $1: 2,000$ ) production ${ }^{37,65,66}$. Intracellular mucin content levels and the extent of mucous plugging were examined by systematic uniform random sampling ${ }^{67}$. Stained slides were scanned by two blinded investigators using an Olympus BX63 microscope and cellSens software (Olympus USA, Center Valley, PA). At least five axial bronchi per mouse were analysed. For non-axial 'bronchiolar' airways, samples were analysed by selection of every fifth $\times 20$ image in a continuous $x$ - $/ y$ map of all lung samples per slide. Mucin and airway area were calculated using point-grid counting. Basement membrane length and airway circumferences were measured in cellSens. Data were calculated as volume densities (volume per unit surface area of basement membrane) $)^{9,65}$ and as extracellular mucous volume fractions relative to lung and airway volumes (multiplied by 100 to express as \% occlusion) ${ }^{67}$

Statistical analyses. All statistical analyses were performed using GraphPad Prism 5.04 with a $P$ value cutoff of 0.05 considered significant. Inflammation, mucin production and mucin secretion data were compared between groups using unpaired one-way Student's $t$-tests, with corrections made for unequal variances (Welch's), or non-Gaussian distribution (Mann-Whitney) where appropriate. For multiple comparisons, analysis of variance (ANOVA) with a Dunnett post hoc correction or Kruskal-Wallis test for non-Gaussian distribution was used. For dose-response tests, curves were compared using ANOVA for repeated measures, and using ANOVA with a Dunnett post hoc correction to compare slopes after linear regression against log-transformed $\mathrm{MCh}$ doses. Both analyses of doseresponse tests provided congruent results. Sample sizes were chosen based on the numbers of WT saline versus allergic mice required to achieve a statistically significant difference.

\section{References}

1. Fanta, C. H. Asthma. New Engl. J. Med. 360, 1002-1014 (2009).

2. Bosse, Y., Riesenfeld, E. P., Pare, P. D. \& Irvin, C. G. It's not all smooth muscle: non-smooth-muscle elements in control of resistance to airflow. Annu. Rev. Physiol. 72, 437-462 (2010).

3. Wagers, S. S. et al. Extravascular fibrin, plasminogen activator, plasminogen activator inhibitors, and airway hyperresponsiveness. J. Clin. Invest. 114, 104-111 (2004).

4. Fahy, J. V. \& Dickey, B. F. Airway mucus function and dysfunction. New Engl J. Med. 363, 2233-2247 (2010).

5. Lundblad, L. K. et al. Airway hyperresponsiveness in allergically inflamed mice: the role of airway closure. Am. J. Respir. Crit. Care Med. 175, 768-774 (2007).

6. McCarthy, D. \& Milic-Emili, J. Closing volume in asymptomatic asthma. Am. Rev. Respir. Dis. 107, 559-570 (1973).

7. Wagers, S. S. et al. Intrinsic and antigen-induced airway hyperresponsiveness are the result of diverse physiological mechanisms. J. Appl. Physiol. (1985) 102, 221-230 (2007).

8. Wagers, S., Lundblad, L. K., Ekman, M., Irvin, C. G. \& Bates, J. H. The allergic mouse model of asthma: normal smooth muscle in an abnormal lung? J. Appl. Physiol. 96, 2019-2027 (2004).

9. Evans, C. M. et al. Mucin is produced by clara cells in the proximal airways of antigen-challenged mice. Am. J. Respir. Cell Mol. Biol. 31, 382-394 (2004).

10. Woodruff, P. G. et al. T-helper type 2-driven inflammation defines major subphenotypes of asthma. Am. J. Respir. Crit. Care Med. 180, 388-395 (2009).

11. Fahy, J. V. Goblet cell and mucin gene abnormalities in asthma. Chest 122, 320S-326S (2002).

12. Ordonez, C. L. et al. Mild and moderate asthma is associated with airway goblet cell hyperplasia and abnormalities in mucin gene expression. Am. J. Respir. Crit. Care Med. 163, 517-523 (2001). 
13. Takeyama, K., Fahy, J. V. \& Nadel, J. A. Relationship of epidermal growth factor receptors to goblet cell production in human bronchi. Am. J. Respir. Crit. Care Med. 163, 511-516 (2001).

14. Zudhi Alimam, M. et al. Muc-5/5ac mucin messenger RNA and protein expression is a marker of goblet cell metaplasia in murine airways. Am. J. Respir. Cell Mol. Biol. 22, 253-260 (2000).

15. Kuyper, L. M. et al. Characterization of airway plugging in fatal asthma. Am. J. Med. 115, 6-11 (2003).

16. Aikawa, T., Shimura, S., Sasaki, H., Ebina, M. \& Takishima, T. Marked goblet cell hyperplasia with mucus accumulation in the airways of patients who died of severe acute asthma attack. Chest 101, 916-921 (1992).

17. Dunnill, M. S. The pathology of asthma, with special reference to changes in the bronchial mucosa. J. Clin. Pathol. 13, 27-33 (1960).

18. The Collaborative Study on the Genetics of Asthma. A genome-wide search for asthma susceptibility loci in ethnically diverse populations. The Collaborative Study on the Genetics of Asthma (CSGA). Nat. Genet. 15, 389-392 (1997).

19. Sheehan, J. K., Richardson, P. S., Fung, D. C., Howard, M. \& Thornton, D. J. Analysis of respiratory mucus glycoproteins in asthma: a detailed study from a patient who died in status asthmaticus. Am. J. Respir. Cell Mol. Biol. 13, 748-756 (1995).

20. Innes, A. L. et al. Ex vivo sputum analysis reveals impairment of proteasedependent mucus degradation by plasma proteins in acute asthma. Am. J. Respir. Crit. Care Med. 180, 203-210 (2009).

21. Singer, M. et al. A MARCKS-related peptide blocks mucus hypersecretion in a mouse model of asthma. Nat. Med. 10, 193-196 (2004).

22. Foster, W. M. et al. MARCKS-related peptide modulates in vivo the secretion of airway Muc5ac. Am. J. Physiol. Lung Cell. Mol. Physiol. 299, L345-L352 (2010).

23. Kirkham, S., Sheehan, J. K., Knight, D., Richardson, P. S. \& Thornton, D. J. Heterogeneity of airways mucus: variations in the amounts and glycoforms of the major oligomeric mucins MUC5AC and MUC5B. Biochem. J. 361, 537-546 (2002).

24. Young, H. W. et al. Central role of Muc5ac expression in mucous metaplasia and its regulation by conserved 5' elements. Am. J. Respir. Cell Mol. Biol. 37, 273-290 (2007)

25. Roy, M. G. et al. Muc5b is required for airway defence. Nature 505, 412-416 (2014).

26. Koeppen, M. et al. Detrimental role of the airway mucin Muc5ac during ventilator-induced lung injury. Mucosal Immunol. 6, 762-775 (2013).

27. Busse, W. W. \& Lemanske, Jr. R. F. Asthma. New Engl. J. Med. 344, 350-362 (2001).

28. Holt, P. G. \& Sly, P. D. Th2 cytokines in the asthma late-phase response. Lancet 370, 1396-1398 (2007).

29. Corren, J. et al. Lebrikizumab treatment in adults with asthma. New Engl. J. Med. 365, 1088-1098 (2011).

30. Wills-Karp, M. et al. Interleukin-13: central mediator of allergic asthma. Science 282, 2258-2261 (1998).

31. Grunig, G. et al. Requirement for IL-13 independently of IL-4 in experimental asthma. Science 282, 2261-2263 (1998).

32. Hershey, G. K. IL-13 receptors and signaling pathways: an evolving web. $J$. Allergy Clin. Immunol. 111, 677-690 (2003).

33. Perkins, C. et al. Selective stimulation of IL-4 receptor on smooth muscle induces airway hyperresponsiveness in mice. J. Exp. Med. 208, 853-867 (2011).

34. Kuperman, D. A. et al. Direct effects of interleukin-13 on epithelial cells cause airway hyperreactivity and mucus overproduction in asthma. Nat. Med. 8, 885-889 (2002).

35. Zhen, G. et al. IL-13 and epidermal growth factor receptor have critical but distinct roles in epithelial cell mucin production. Am. J. Respir. Cell Mol. Biol. 36, 244-253 (2007)

36. Takeyama, K. et al. Epidermal growth factor system regulates mucin production in airways. Proc. Natl Acad. Sci. USA 96, 3081-3086 (1999).

37. Hasnain, S. Z. et al. Muc5ac: a critical component mediating the rejection of enteric nematodes. J. Exp. Med. 208, 893-900 (2011).

38. Kheradmand, F. et al. A protease-activated pathway underlying Th cell type 2 activation and allergic lung disease. J. Immunol. 169, 5904-5911 (2002).

39. Takeda, K., Haczku, A., Lee, J. J., Irvin, C. G. \& Gelfand, E. W. Strain dependence of airway hyperresponsiveness reflects differences in eosinophil localization in the lung. Am. J. Physiol. Lung Cell. Mol. Physiol. 281, L394-L402 (2001).

40. Kim, K. C. \& Lee, B. C. P2 purinoceptor regulation of mucin release by airway goblet cells in primary culture. Br. J. Pharmacol. 103, 1053-1056 (1991).

41. Lethem, M. I. et al. Nucleotide regulation of goblet cells in human airway epithelial explants: normal exocytosis in cystic fibrosis. Am. J. Respir. Cell Mol. Biol. 9, 315-322 (1993).

42. Govindaraju, V., Martin, J. G., Maghni, K., Ferraro, P. \& Michoud, M. C. The effects of extracellular purines and pyrimidines on human airway smooth muscle cells. J. Pharmacol. Exp. Ther. 315, 941-948 (2005).
43. Johansson, M. E. et al. The inner of the two Muc2 mucin-dependent mucus layers in colon is devoid of bacteria. Proc. Natl Acad. Sci. USA 105 15064-15069 (2008).

44. Lundgren, J. D. \& Vestbo, J. The pathophysiological role of mucus production in inflammatory airway diseases. Respir. Med. 89, 315-316 (1995).

45. Israel, E. et al. Use of regularly scheduled albuterol treatment in asthma: genotype-stratified, randomised, placebo-controlled cross-over trial. Lancet 364, 1505-1512.

46. Shan, M. et al. Mucus enhances gut homeostasis and oral tolerance by delivering immunoregulatory signals. Science 342, 447-453 (2013).

47. Hogg, J. C. The pathology of asthma. Clin. Chest Med. 5, 567-571 (1984).

48. O'Riordan, T. G., Zwang, J. \& Smaldone, G. C. Mucociliary clearance in adult asthma. Am. Rev. Respir. Dis. 146, 598-603 (1992).

49. Innes, A. L. et al. The $\mathrm{H}$ antigen at epithelial surfaces is associated with susceptibility to asthma exacerbation. Am. J. Respir. Crit. Care Med. 183, 189-194 (2011).

50. Verdugo, P. Supramolecular dynamics of mucus. Cold Spring Harb. Perspect. Med. 2, a009597 (2012).

51. Daviskas, E. et al. Inhalation of hypertonic saline aerosol enhances mucociliary clearance in asthmatic and healthy subjects. Eur. Respir. J. 9, 725-732 (1996).

52. Falliers, C. J. \& Cato, A. Controlled trial of bronchodilator-mucolytic aerosols, combined and separate. Ann. Allergy 40, 77-83 (1978).

53. Rao, S., Wilson, D. B., Brooks, R. C. \& Sproule, B. J. Acute effects of nebulization of $\mathrm{N}$-acetylcysteine on pulmonary mechanics and gas exchange. Am. Rev. Respir. Dis. 102, 17-22 (1970).

54. Tam, J., Nash, E. F., Ratjen, F., Tullis, E. \& Stephenson, A. Nebulized and oral thiol derivatives for pulmonary disease in cystic fibrosis. Cochrane Database Syst. Rev. 7, CD007168 (2013).

55. Gillissen, A. et al. Nacystelyn, a novel lysine salt of $\mathrm{N}$-acetylcysteine, to augment cellular antioxidant defence in vitro. Respir. Med. 91, 159-168 (1997).

56. Suk, J. S. et al. N-acetylcysteine enhances cystic fibrosis sputum penetration and airway gene transfer by highly compacted DNA nanoparticles. Mol. Ther. 19, 1981-1989 (2011).

57. Jayaraman, S., Song, Y. \& Verkman, A. S. Airway surface liquid pH in welldifferentiated airway epithelial cell cultures and mouse trachea. Am. J. Physiol. Cell Physiol. 281, C1504-C1511 (2001).

58. Alevy, Y. G. et al. IL-13-induced airway mucus production is attenuated by MAPK13 inhibition. J. Clin. Invest. 122, 4555-4568 (2012).

59. Woodruff, P. G. et al. Safety and efficacy of an inhaled epidermal growth factor receptor inhibitor (BIBW 2948 BS) in chronic obstructive pulmonary disease. Am. J. Respir. Crit. Care Med. 181, 438-445 (2010).

60. Tsao, P. N. et al. Notch signaling prevents mucous metaplasia in mouse conducting airways during postnatal development. Development 138, 3533 3543 (2011).

61. Chen, G. et al. SPDEF is required for mouse pulmonary goblet cell differentiation and regulates a network of genes associated with mucus production. J. Clin. Invest. 119, 2914-2924 (2009).

62. Park, K. S. et al. SPDEF regulates goblet cell hyperplasia in the airway epithelium. J. Clin. Invest. 117, 978-988 (2007).

63. Schroeder, B. W. et al. AGR2 is induced in asthma and promotes allergeninduced mucin overproduction. Am. J. Respir. Cell Mol. Biol. 47, 178-185 (2012).

64. Foster, M. W., Yang, Z., Potts, E. N., Michael Foster, W. \& Que, L. G. Snitrosoglutathione supplementation to ovalbumin-sensitized and -challenged mice ameliorates methacholine-induced bronchoconstriction. Am. J. Physiol. Lung Cell. Mol. Physiol. 301, L739-L744 (2011).

65. Piccotti, L., Dickey, B. F. \& Evans, C. M. Assessment of intracellular mucin content in vivo. Methods Mol. Biol. 842, 279-295 (2012).

66. Roy, M. G. et al. Mucin production during prenatal and postnatal murine lung development. Am. J. Respir. Cell Mol. Biol. 44, 755-760 (2011).

67. Hsia, C. C., Hyde, D. M., Ochs, M. \& Weibel, E. R. An official research policy statement of the American Thoracic Society/European Respiratory Society: standards for quantitative assessment of lung structure. Am. J. Respir. Crit. Care Med. 181, 394-418 (2010).

\section{Acknowledgements}

We thank G. Francis, F. Benavides and J. Parker-Thornburg for their assistance in generating mutant and congenic mice. Discussions with Dr W.M. Foster of the Duke University Medical Center were helpful in the design of our aerosol system. This work was supported by the National Institutes of Health Grants R01HL080396 and R21ES023384 (C.M.E.); R21HL120770 (D.A.S.); UH2HL123442 (C.M.E. and D.A.S.); R01HL097000 (B.F.D.); R01HL070952 (M.R.B.); R01DK097075, R01HL092188, R01HL098294 and R01HL119837 (H.K.E.); P01HL114457 (M.R.B. and H.K.E.); R01HL109517 (W.J.J.); and grants by the American Heart Association 14GRNT19990040 (C.M.E.) and the Crohn's and Colitis Foundation of America (H.K.E.). T.A.W. was supported by the intramural research program at the National Institute of Allergy and Infectious Diseases/National Institutes of Health. Additional support was provided by National Institutes of Health Cancer Center Support Grants P30CA016672 (M.D. 
Anderson Cancer Center, including the Research Animal Support Facility-Smithville, Animal Genetics Services) and P30CA046934 (University of Colorado).

\section{Author contributions}

D.S.R., F.T., D.R.L., A.A.F., D.N.H., M.A.McG., M.M.McE., O.W.W., E.S. and M.G.R. bred animals, performed exposures, performed studies of lung function, inflammation and mucin production/secretion. K.N.K., T.A.W., H.K.E., M.R.B., M.J.T., D.A.S. and B.F.D. assisted in study design and data analysis. C.M.E. conceptualized the study, performed experiments and wrote the manuscript.

\section{Additional information}

Supplementary Information accompanies this paper at http://www.nature.com/ naturecommunications

Competing financial interests: The authors declare no competing financial interests.

Reprints and permission information is available online at http://npg.nature.com/ reprintsandpermissions/

How to cite this article: Evans, C. M. et al. The polymeric mucin Muc5ac is required for allergic airway hyperreactivity. Nat. Commun. 6:6281 doi: 10.1038/ncomms7281 (2015). 